findet ${ }^{8}$. Dieser Effekt steht ganz deutlich in Zusammenhang mit dem Übergang von ausgeprägter polarer zu ausgeprägter metallischer Bindung. Auch das ist ein Hinweis dafür, daß die Bindung der $\mathrm{DO}_{3^{-}}\left(\mathrm{BiF}_{3^{-}}\right)$-Strukturen im wesentlichen polarer Natur ist.

Die $\mathrm{DO}_{3}$-Struktur tritt auf, wenn große Anionen (Kationen) mit kleinen Kationen (Anionen) im Ver- hältnis $1: 3$ vorliegen. Dabei bilden die großen Ionen eine dichte, kubisch-flächenzentrierte Packung, in deren Tetraeder- und Oktaederlücken die kleinen Ionen eingelagert sind. Es ist verständlich, daß mit dieser Packung der tiefste Energiezustand zu erreichen ist.

Herrn Dr. J. P. Suchet (Bellevue, Frankreich) danke ich für wertvolle Diskussionen.

\section{Ferromagnetische Desakkommodation durch Zwischengitteratome in neutronenbestrahltem Nickel}

\author{
Von H. Kronmüller, A. Seeger und P. Schiller \\ Max-Planck-Institut für Metallforschung, Stuttgart, \\ und Institut für theoretische und angewandte Physik \\ der Technischen Hochschule Stuttgart \\ (Z. Naturforschg. 15 a, 740-741 [1960] ; eingegangen am 19. Juli 1960)
}

An anderer Stelle ${ }^{1}$ haben wir gezeigt, daß die bei der plastischen Verformung von Nickel entstandenen Zwischengitteratome durch einen Art Sкоек-Effekt nachgewiesen werden können. Dieser beruht auf folgendem: Die stabile Lage eines Zwischengitteratoms im kubischflächenzentrierten Gitter ist nicht die kubisch-symmetrische Lage in der Mitte des Elementarwürfels, sondern die sog. Hantellage (,intersticialcy" ${ }^{2}$ ), in der das Zwischengitteratom und eines seiner nächsten Nachbaratome längs der Richtung der Würfelkante verschoben sind und infolge der resultierenden tetragonalen Symmetrie eine der $\langle 100\rangle$-Richtungen vor den beiden anderen ausgezeichnet ist. Die Verteilung der Hanteln auf die drei $\langle 100\rangle$-Richtungen hängt von der mechanischen (oder auch magnetostriktiven) Spannung $a b$; ändert sich diese, so tritt infolge der allmählichen Änderung dieser Verteilung ein Relaxationsvorgang ein, der sich durch mechanische bzw. magnetische Messungen verfolgen läßt. Die Relaxationszeit $\tau$ ist bestimmt durch die Häufigkeit, mit der die Hanteln unter dem Einfluß thermischer Schwankungen ihre Orientierung ändern können.

Die frühere ${ }^{1}$ und die jetzige Untersuchung wurden an Nickel ausgeführt, weil erstens wegen der verhältnismäßig großen Wanderungsenergie der Zwischengitteratome ( $W=1,02 \mathrm{eV}$ - siehe unten) diese bei Raumtemperatur nur sehr langsam ausheilen und weil zweitens der erwähnte Relaxationseffekt sich als Nachwirkung (Desakkommodation) der ferromagnetis.chen Suszeptibilität $\mu$ besonders genau messen läßt ${ }^{*}$. Da jedoch theoretische Untersuchungen für Kupfer ${ }^{3}$ in guter Übereinstimmung mit den Befunden an Nickel sind (die Wanderungsaktivierungsenergie - gerechnet als Energiedifferenz zwischen der kubisch-symmetrischen Lage und der Hantellage - ergab sich zu $W \approx 0,6 \mathrm{eV}$, ent-

1 A. Seeger, P. Schiller u. H. Kronmüller, Phil. Mag. 5, im Druck.

2 H. B. Huntington u. F. Seitz, Phys. Rev. 61, 315 [1942].

* Der entsprechende Nachwirkungseffekt als Folge der SNoEk-Relaxation der Kohlenstoffatome im $\alpha$-Fe ist als Richternachwirkung bekannt. sprechend dem Unterschied in den Schmelztemperaturen), glauben wir unsere Ergebnisse auch auf die Edelmetalle übertragen zu können.

Unsere Messungen wurden an Karbonyl-Nickel ausgeführt, das im Forschungsreaktor München mit einer integrierten Flußdichte $n v t=4 \cdot 10^{17} \mathrm{~cm}^{-2}$ von schnellen Neutronen mit Energien oberhalb $0,1 \mathrm{MeV}$ bestrahlt wurde und während der 4 Stunden dauernden Bestrahlung auf etwa $25{ }^{\circ} \mathrm{C}$ gekühlt worden war. Während der Lagerungszeit nach der Bestrahlung wurden die Proben entweder mit flüssiger Luft oder mit fester Kohlensäure gekühlt, um das Ausheilen der Zwischengitteratome zu verhindern. Die Suszeptibilitätsmessungen wurden mit der von Gerstner ${ }^{4}$ beschriebenen Apparatur durchgeführt.

Abb. 1 zeigt die gemessenen Relaxationszeiten als Funktion der reziproken absoluten Temperatur. Die offenen Quadrate wurden am reaktorbestrahlten Mate-

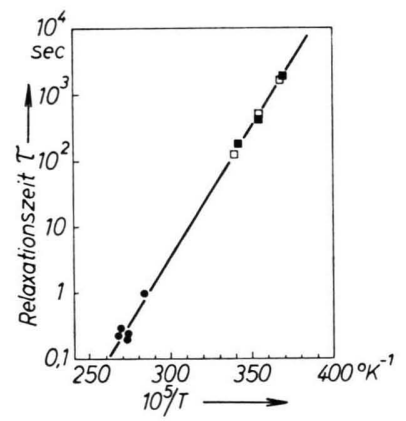

Abb. 1. Die Relaxationszeit (in logarithmischer Auftragung) als Funktion der reziproken absoluten Temperatur $T$. $\bullet$ : innere Reibung nach plastischer Verformung. a: magnetische Nachwirkung nach plastischer Verformung. $\square$ : magnetische Nachwirkung nach Neutronenbestrahlung.

rial durch die ferromagnetische Nachwirkung gemessen. Außerdem haben wir die z. Tl. schon mitgeteilten Messungen mit dem Torsionspendel an plastisch verformten Proben $^{1}$ (volle Kreise) und Desakkommodationsmessungen an verformten Proben ** (volle Quadrate) mit eingetragen. Wie man sieht, gehorchen die Messungen nach Verformung und nach Bestrahlung einem gemeinsamen

${ }^{3}$ A. Seeger, E. Mann u. R. v. Jan, Veröffentlichung demnächst.

4 D. Gerstner, Dissertation, Stuttgart 1960.

** Die eingetragenen Meßpunkte stammen von H. JäGER und sind neueren Datums als die früher ${ }^{1}$ mitgeteilten. 


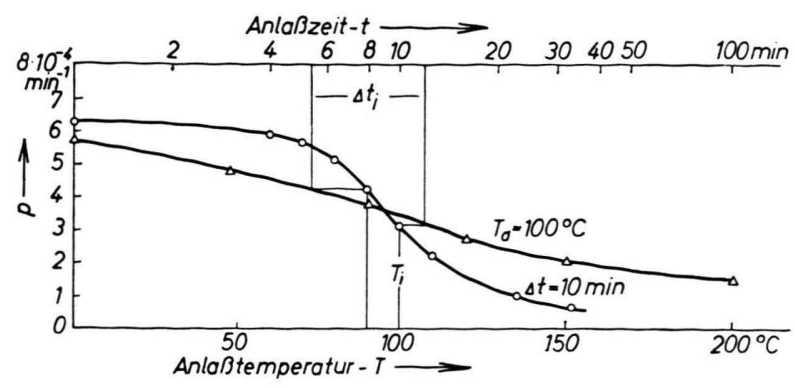

Abb. 2. Erholung der magnetischen Nachwirkung bei reaktorbestrahltem Nickel. Als Ordinate ist die Geschwindigkeit der Änderung der relativen Permeabilität $p=\frac{\mathrm{d}}{\mathrm{d} t} \frac{\mu(t)-\mu(\infty)}{\mu(\infty)}$ bei $10{ }^{\circ} \mathrm{C}$ nach 13 min aufgetragen. $\triangle$ : Isotherme Erholung bei $T_{\mathrm{a}}=100^{\circ} \mathrm{C}$. $\bigcirc$ : Isochrone Erholung mit Haltezeiten $\Delta t=10 \mathrm{~min}$. Die Brinkman-Meechansche Konstruktion zur Ermittlung der Aktivierungsenergie ist an einem Beispiel dargestellt.

\section{Gesetz}

$$
\tau=\tau_{0} \exp (Q / k T) .
$$

Dies ist im Einklang mit unserer Interpretation, da ja Zwischengitteratome sowohl bei plastischer Verformung als auch bei Bestrahlung gebildet werden. Aus Abb. 1 entnimmt man für die Aktivierungsenergie des Relaxationsvorganges

$$
Q=0,81 \pm 0,01 \mathrm{eV}
$$

und für die Zeitkonstante bei unendlich hoher Temperatur

$$
\tau_{0}=2,6 \cdot 10^{-12} \mathrm{sec} .
$$

Abb. 2 zeigt die Ergebnisse von Anlaßexperimenten, und zwar je eine Isochrone (gleiche Haltezeiten $\Delta t$ bei aufeinanderfolgenden Temperaturen $T_{\mathrm{i}}$ ) und eine Isotherme (zeitlicher Verlauf bei konstanter Temperatur $T_{\mathrm{a}}$ ). Kombiniert man in der angedeuteten Weise die beiden Messungen miteinander, so bekommt man nach MeEchan und Brinkman ${ }^{5}$ in der Darstellung der Abb. 3 dann und nur dann eine Gerade, wenn es sich um einen Prozeß mit einheitlicher Aktivierungsenergie handelt. Der Anstieg gegen $(k T)^{-1}$ ist gleich der Aktivierungsenergie $W$ des Ausheilprozesses. Diese ergibt sich zu

$$
W=1,02 \pm 0,03 \mathrm{eV} \text {. }
$$

Verwenden wir die in Abb. 2 dargestellte Isotherme zur Bestimmung der Reaktionsordnung der Erholung, so ergibt sich für die Größe $p^{-1}$ ein im Rahmen der Meßgenauigkeit linearer Verlauf mit der Anlaßdauer. Dies weist auf eine Reaktion 2. Ordnung hin, welche

5 J. C. Meechan u. J. A. Brinkman, Phys. Rev. 103, 1193 [1956].

6 A. Sosin u. J. A. Brinkman, Acta Met. 7, 478 [1959].

7 J. A. Brinkman, C. E. Dixon u. J. C. Meechan, Acta Met. 2, 38 [1954].

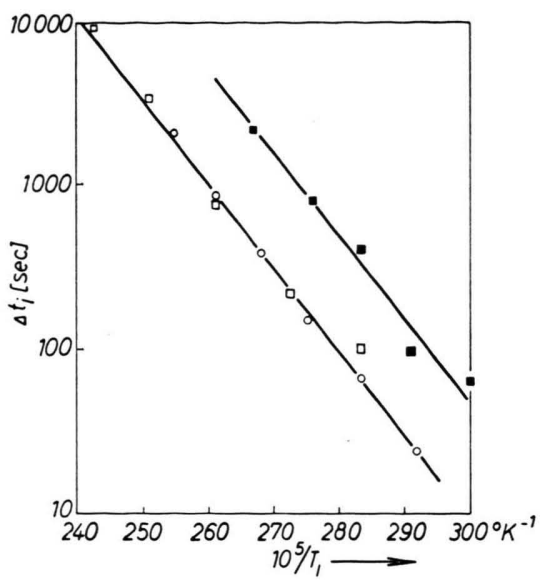

Abb. 3. Bestimmung der Aktivierungsenergie des Ausheilvorgangs nach dem Brinkman-Meechan-Verfahren. Die Aktivierungsenergie ist gegeben durch $W=-\mathrm{d} \ln \left(\Delta t_{\mathrm{i}}\right) / \mathrm{d}\left(1 / T_{\mathrm{i}}\right)$. $\bigcirc$ : Erholung der magnetischen Nachwirkung nach Reaktorbestrahlung (entnommen aus Abb. 2). घ: Erholung des elektrischen Widerstands nach plastischer Verformung (Sosin und Brinkman). $\square$ : Erholung des elektrischen Widerstands nach Elektronenbestrahlung (Sosin und Brinkman). Die verschiedenen Absoluthöhen der Kurven rühren von verschieden gewählten Anlaßtemperaturen und Haltezeiten $\Delta t$ her.

darauf beruht, daß die Zwischengitteratome bevorzugt durch Rekombination mit Leerstellen ausheilen.

Die Aktivierungsenergie $W$ stimmt mit der von Sosin und Brinkman ${ }^{6}$ aus dem Ausheilen des elektrischen Widerstandes von elektronenbestrahltem und von kaltverformtem Nickel in der sog. Stufe III ermittelten und ebenfalls dem Ausheilen der Zwischengitteratome zugeschriebenen sehr gut überein. Unsere Untersuchungen scheinen uns den Beweis für die Richtigkeit dieser Interpretation ${ }^{7,8,9,10,5}$ und damit auch eine Klärung der diesbezüglichen Fragen in der Theorie der Strahlungsschädigung der Edelmetalle erbracht zu haben. Wegen der verschiedenen Herkunft und Vorbehandlung des in den besprochenen Experimenten verwendeten Nickels scheint es uns ausgeschlossen, da $\beta$ Verunreinigungen bei diesen Erscheinungen eine wesentliche Rolle spielen.

Wir danken den zuständigen Herrn des Forschungsreaktors München, insbesondere Herrn Dr. Schilling, für die Durchführung der Bestrahlung und das tatkräftige Eingehen auf unsere Wünsche. Die Meßapparatur war dankenswerterweise von der De u t s chen For $\mathrm{schungsgemeinschaft}$ zur Verfügung gestellt worden.

8 A. SEEGER, Z. Naturforschg. 10 a, 251 [1955].

9 A. SeEger, Z. Naturforschg. 13 a, 54 [1958].

10 A. SEEgER, Proc. Second United Nations International Conference on the Peaceful Uses of Atomic Energy 6, 250, Genf 1958 . 


\section{Mikrowellenspektrum, Struktur und Hinderungspotential des Dimethylsulfids}

Von H. D. Rudolph, H. Dreizler und W. Maier

Institut für physikalische Chemie der Universität Freiburg/Br.

(Z. Naturforschg. 15 a, 742 [1960]; eingegangen am 1. Juli 1960)

Wir haben das Mikrowellenspektrum des Dimethylsulfids $\left(\mathrm{CH}_{3}\right)_{2} \mathrm{~S}$ aufgenommen und die Struktur des Moleküls sowie das Hinderungspotential der beiden partiell drehbaren $\mathrm{CH}_{3}$-Gruppen bestimmt.

Es wurde ein mit Carcinotrons (CSF) als Strahlungsquellen bestückter Mikrowellen-Spektrograph mit 30 $\mathrm{kHz}$-Sтаякеffekt-Modulation benutzt, der den Frequenzbereich von 8 bis $37 \mathrm{GHz}$ lückenlos überstreicht. Zur Frequenzmessung dienten von der Mikrowellen-Frequenzdekade FD 3 (Schomand) abgeleitete Frequenzmarken (s. auch Abb. 1) ; die Genauigkeit der in Tab. 1 angegebenen Linienfrequenzen beträgt $\pm 0,05 \mathrm{MHz}$.

\begin{tabular}{|c|c|c|}
\hline $\begin{array}{c}\text { Zuordnung } \\
J_{\mathrm{K}_{-} \mathrm{K}_{+} \rightarrow J^{\prime} \mathrm{K}_{-}^{\prime} \mathrm{K}_{+}^{\prime}}\end{array}$ & $\begin{array}{c}\text { gemessene Frequenz } \\
\mathrm{MHz}\end{array}$ & $\begin{array}{c}\text { berechnete Frequenz } \\
\mathrm{MHz}\end{array}$ \\
\hline $1_{01} \rightarrow 1_{10}$ & $12091,98 \pm 0,05$ & 12091,59 \\
$2_{02} \rightarrow 2_{11}$ & 14237,88 & 14237,33 \\
$1_{11} \rightarrow 2_{02}$ & 16246,26 & 16246,65 \\
$0_{00} \rightarrow 1_{11}$ & 23527,52 & 23527,19 \\
$2_{11} \rightarrow 2_{20}$ & 30807,32 & 30807,74 \\
$1_{01} \rightarrow 2_{12}$ & 34963,20 & 34962,79 \\
$2_{12} \rightarrow 2_{21}$ & 36274,51 & 36274,78 \\
\hline
\end{tabular}

Tab. 1. Die zur Strukturbestimmung herangezogenen Absorptionslinien von $\left(\mathrm{C}^{12} \mathrm{H}_{3}\right)_{2} \mathrm{~S}^{32}$ mit $J \leqq 2$ im Frequenzbereich bis $37000 \mathrm{MHz}$.

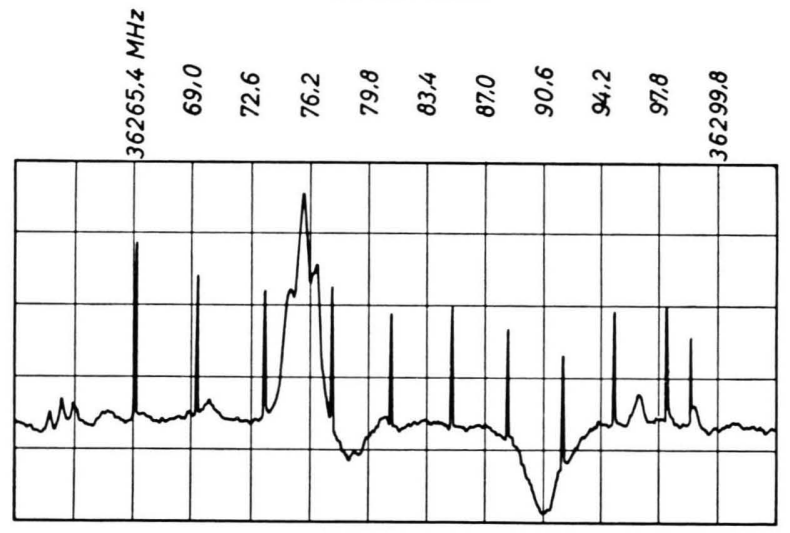

Abb. 1. Linie $2_{12} \rightarrow 2_{21}$ des $\left(\mathrm{C}^{12} \mathrm{H}_{3}\right)_{2} \mathrm{~S}^{32}$ bei $36274,5 \mathrm{MHz}$ Triplettaufspaltung. Carcinotron CO 1308, Druck ca. 10-2 Torr, Stark-Modulationsfeldstärke $1990 \mathrm{~V} / \mathrm{cm}$, Verstärkerzeitkonstante $1 \mathrm{sec}$, Schreibgeschwindigkeit $70 \mathrm{kHz} / \mathrm{sec}$, Detektor $1 \mathrm{~N} 53$, bezifferte Frequenzmarken in $\mathrm{MHz}$.

Wir haben in dem genannten Frequenzbereich eine große Anzahl von Linien gefunden und davon $29 \mathrm{zu}$ geordnet. Die Modellvorstellung des Moleküls als quasistarrer, asymmetrischer Kreisel erwies sich als hinreichend genau für diese Zuordnungsaufgabe. Der Einfluß der beiden behindert drehbaren $\mathrm{CH}_{3}$-Gruppen bewirkt, daß die beim starren Kreiselmolekül einfachen Rotationslinien in enge, gleichabständige Tripletts auf- spalten, von denen einige getrennt werden konnten. Die Linie bei $36274,5 \mathrm{MHz}$ zeigt mit $\pm 0,72 \mathrm{MHz}$ die größte der gefundenen Triplettaufspaltungen (Bild 1). Für die Linien mit nur durch Schultern angedeuteter Aufspaltung war nach theoretischen Überlegungen tatsächlich eine geringere Aufspaltung zu erwarten. Andere Autoren ${ }^{1}$ haben bei Molekülen mit zwei behindert drehbaren Gruppen eine aus den genannten Tripletts durch Verdopplung eines Triplettpartners hervorgehende Quartettstruktur der Rotationslinien festgestellt. Wir hingegen konnten wegen des recht hohen Hinderungspotentials und der speziellen Struktur des Moleküls nur Tripletts beobachten. Deren Intensitätsverhältnisse entsprachen mit $3: 8: 3$ für Rotationsspezies $A$ und $B_{x}$ und mit $5: 8: 5$ für Rotationsspezies $B_{y}$ und $B_{z}$ den theoretischen Erwartungen ${ }^{1,2}$.

Wir haben zur Strukturbestimmung nur Linien bis $J=2$ herangezogen, um die Zentrifugalaufweitung des Moleküls nicht berücksichtigen zu müssen. Die Rotationskonstanten sind: $A=17809,39 \mathrm{MHz}, B=7621,00 \mathrm{MHz}$ $C=5717,80 \mathrm{MHz}$; der Asymmetrieparameter beträgt $\varkappa=-0,6852$. Die Strukturdaten des Moleküls haben wir aus diesen Konstanten unter den folgenden naheliegenden Voraussetzungen bestimmt:

(a) Die Methylgruppen sind symmetrisch, $\Varangle \mathrm{HCH}$ ist der Tetraederwinkel.

(b) Die Symmetrieachse der $\mathrm{CH}_{3}$-Gruppe verläuft koaxial mit der SC-Bindung.

Ein etwaiger Knickwinkel zwischen $\mathrm{CH}_{3}$-Symmetrieachse und SC-Bindung läßt sich nur durch Hinzunahme von Isotopenmolekülen bestimmen, wie wir es auch planen. Die nach diesen Annahmen noch verbleibenden Strukturdaten ergaben sich zu

$$
\Varangle \mathrm{CSC}=98^{\circ} 58^{\prime}
$$

Abstand $\mathrm{SC}=1,809 \AA \AA \quad$ Abstand $\mathrm{CH}=1,083 \AA \AA$.

Die Fehler dieser Bestimmungen wären bei strenger Gültigkeit der Voraussetzungen (a) und (b) sehr klein $\left(0,5^{\prime}\right.$ bzw. $\left.0,001 \AA\right)$, bei kritischer Würdigung ihrer Gültigkeit (Vergleich mit ähnlich gelagerten Fällen ${ }^{1,2}$ ) lassen sie sich abschätzen zu Fehler $\Varangle$ CSC ca. $\pm 0,3^{\circ}$, Fehler Abstand SC ca. $\pm 0,005 \AA \AA$, Fehler Abstand CH ca. $\pm 0,01 \AA$.

Die Theorie von Swalen und Costain ${ }^{1}$ gestattet die näherungsweise Bestimmung des Hinderungspotentials für die Drehung der $\mathrm{CH}_{3}$-Gruppen aus der Triplettaufspaltung der Linien auf dem Wege über die für die verschiedenen Triplettpartner verschiedenen effektiven Rotationskonstanten. Dabei benutzten wir die Tabellen von $\mathrm{Herschbach}^{3}$. Die genannte Theorie liefert das Hinderungspotential $\mathrm{zu}$ :

$$
V=730 \pm 30 \mathrm{~cm}^{-1}=2,09 \pm 0,1 \mathrm{kcal} / \mathrm{Mol} \text {. }
$$

Der angegebene Fehler versucht, etwaige Ungenauig. keiten in den Annahmen (a) und (b) bereits zu berücksichtigen.

Wir danken der Deutschen Forschungs g e meinsch a f t für personelle und sachliche Unterstützung und Herrn Prof. Dr. R. Mecke für die freundliche Überlassung der Hilfsmittel des Instituts.

1 J.D. Swalen u. C. C. Costain, J. Chem. Phys. 31, 1562 [1959]. 2 P. H. Kasai u. R. J. Myers, J. Chem. Phys. 30, 1096 [1959].

3 D. R. Herschbach, J. Chem. Phys. 31, 91 [1959]. 